\title{
Characterization of the inflammatory and apoptotic cells in the aortas of patients with ascending thoracic aortic aneurysms and dissections
}

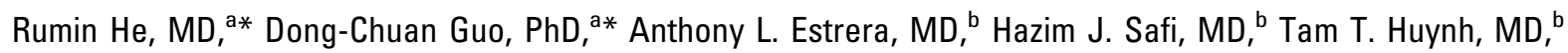
Zhengnan Yin, MD, PhD, ${ }^{a}$ Shi-Nian Cao, MD, ${ }^{c}$ Jing Lin, MD, ${ }^{a}$ Thomas Kurian, ${ }^{a}$ L. Maximillian Buja, MD, ${ }^{c}$ Yong-Jian Geng, MD, PhD, and Dianna M. Milewicz, MD, PhD ${ }^{\mathrm{a}}$

Supplemental material is available online.
From the Departments of Internal Medicine, ${ }^{\mathrm{a}}$ Cardiovascular Surgery, ${ }^{\mathrm{b}}$ and $\mathrm{Pa}-$ thology, ${ }^{\mathrm{c}}$ The University of Texas Medical School at Houston, Houston, Tex.

This work was support by grants RO1 HL62594 (D.M.M.), RO1 HL59249 (Y.J.G.), RO1 HL09509 (Y.J.G.), and MO1RR02558 (General Clinical Research Center), and by the Doris Duke Foundation.

Received for publication April 5, 2005; revisions received Aug 11, 2005; accepted for publication Sept 8, 2005.

Address for reprints: Dianna M. Milewicz, $\mathrm{MD}, \mathrm{PhD}$, The University of Texas Medical School at Houston, 6431 Fannin St, MSB 4.202, Houston, TX 77030 (E-mail: Dianna.M.Milewicz@uth.tmc.edu).

*The first two authors contributed equally to this work.

J Thorac Cardiovasc Surg 2006;131:671-8

$0022-5223 / \$ 32.00$

Copyright (C) 2006 by The American Association for Thoracic Surgery

doi:10.1016/j.jtcvs.2005.09.018
Objective: The histopathologic abnormality underlying ascending aortic aneurysm and dissection is medial degeneration, a lesion that is described as the noninflammatory loss of smooth muscle cells and elastic fibers. This study sought to determine whether inflammatory cells are present in medial degeneration and assess any possible contribution of these cells to apoptosis of smooth muscle cells.

Methods: Aortic specimens were obtained from patients undergoing prophylactic surgical repair of an ascending aortic aneurysm $(\mathrm{n}=9)$ and type A dissection $(\mathrm{n}=$ $7)$, along with control patients dying of causes unrelated to aortic disease $(n=5)$. Immunohistochemical staining was performed to evaluate the presence of lymphocytes and macrophages, and markers of apoptosis were assessed in the aortas of patients with ascending aortic aneurysm and dissection.

Results: Immunohistochemical study indicated significantly more $\mathrm{CD} 3^{+}$cells in the aortas of patients with aneurysms or dissections than in control aortas $(P=.020$ and $P=.0022$, respectively). In addition, aortas of patients with aneurysms or dissections had more $\mathrm{CD} 68^{+}$cells $\left(P=.01\right.$ and $P=.005$, respectively). $\mathrm{CD} 3^{+}$cells were localized in the media and surrounding the vasa vasorum in the adventitia. Cells yielding a positive result on in situ terminal transferase-mediated deoxyuridine triphosphate nick end-labeling were found in increased numbers in the aortas of patients with aneurysms or dissections relative to control aortas $(P=.005$ and $P=$ .002 , respectively). Furthermore, Fas and FasL were increased in the aortic samples from patients with aneurysms and dissections relative to control aortas.

Conclusion: The coexistence of inflammatory cells with markers of apoptotic vascular cell death in the media of ascending aortas with aneurysms and type A dissections raises the possibility that activated $\mathrm{T}$ cells and macrophages may contribute to the elimination of smooth muscle cells and degradation of the matrix associated with thoracic aortic aneurysms and dissections.

$\mathrm{T}$ The major disease processes affecting the aorta are aortic aneurysms and dissections, and these diseases account for nearly 16,000 deaths annually. The most common aortic aneurysms are infrarenal abdominal aortic aneurysms (AAAs), followed by ascending thoracic aortic aneurysms (TAAs). TAAs and type A aortic dissections (TADs) are related conditions, as evidenced by the fact that progressive enlargement of a TAA can lead to a TAD in the absence of prophylactic surgical repair. The pathology associated with AAAs is atherosclerosis. In contrast, the most common pathology associated with TAAs and TADs is Erdheim cystic medial necrosis, which is described as a noninflammatory lesion characterized by a triad of smooth muscle cell (SMC) loss, fragmentation of elastic fibers, and accumulation of basophilic ground substance within cell depleted areas 


\section{Abbreviations and Acronyms \\ AAA $=$ abdominal aortic aneurysm \\ SMC $=$ smooth muscle cell \\ TAA = ascending thoracic aortic aneurysm \\ $\mathrm{TAD}=$ type $\mathrm{A}$ aortic dissection \\ TUNEL $=$ terminal transferase-mediated deoxyuridine triphosphate nick-end labeling}

of the medial layer of the aorta. ${ }^{1}$ Because the lesion is neither necrotic nor cystic, more recently the term medial degeneration has been adopted to describe this pathology. Medial degeneration is not just a pathologic condition but is commonly seen in aging aorta and in patients with hypertension. $^{2,3}$ It has been shown that these changes are both qualitatively and quantitatively much greater in patients with TADs. ${ }^{4}$ The lesion is also observed in genetic conditions that predispose individuals toward TAA and TAD, such as Marfan syndrome and familial TAD. ${ }^{5,6}$

Inflammation has been documented in only a small number of causes of human TAAs, such as giant cell arteritis, syphilitic aortitis, and very rare cases of inflammatory aneurysms. ${ }^{7-9}$ In this study, we tested the hypothesis that immune infiltrates are present in the aortas of patients with medial degeneration and that the presence of these inflammatory cells contributes to the local expression of death-promoting mediators in the diseased aortas. We observed that $\mathrm{T}$ lymphocytes and macrophages were present in the aortic media in both TAAs and TADs but occurred in greater numbers in the aortas of patients who had dissections. We observed that the aortas from patients with aneurysms and dissections had higher levels of Fas, FasL, and caspase-3 than did normal aortas, indicating that these pathways are activated in both aortic diseases. With a combination of immunostaining and in situ terminal transferase-mediated deoxyuridine triphosphate nick-end labeling (TUNEL), we detected numerous TUNEL-positive cells that exhibited either SMC markers or CD3 staining, evidence that both $\alpha$-actin-positive and $\mathrm{CD}^{+}$cells are undergoing apoptosis. Thus this study indicates that inflammation is a component of medial degeneration and may contribute to apoptosis and degeneration of the aortic wall during the development of aneurysms and dissections.

\section{Materials and Methods}

\section{Aortic Tissue}

After appropriate informed consent was obtained, full-thickness aortic wall specimens were collected above the aortic valve from patients undergoing elective repair of TAA $(n=9,3$ male and 6 female, average age 62.9 years) and emergency repair of acute TAD ( $\mathrm{n}=7,4$ male and 3 female, average age 59.9 years). All patients had tricuspid aortic valves. None of the patients had a known genetic syndrome causing the aortic disease (eg, Marfan or Turner syndrome) or a family history of
TAA or TAD. Control ascending aortas were obtained from 5 individuals found at autopsy to have died of causes unrelated to aortic disease (2 male and 3 female, average age 57.2 years). Medical histories pertinent to aortic disease were obtained from the patients' medical records (Table E1). Specimens were immediately transferred to the laboratory in a container with cold Waymouth MB 752/1 medium (Invitrogen Corporation, Carlsbad, Calif). Each sample was divided into two parts. The first part was used for histopathologic studies, and the second was used for protein and RNA extraction and for explanting SMCs. The study was approved by the institutional review committee at the University of Texas Health Science Center at Houston and Memorial Hermann Hospital.

\section{SMC Culture}

Aortic layers were separated, minced, and digested for 16 hours in an enzyme mixture containing elastase type III ( $15 \mathrm{U} / \mathrm{mL})$, collagenase type $\mathrm{I}(165 \mathrm{U} / \mathrm{mL})$, and soybean trypsin inhibitor $(0.375$ $\mathrm{mg} / \mathrm{mL}$ ). Digestion was stopped with SmGm2 medium (BioWhittaker Molecular Applications, Rockland, Maine) supplemented with $20 \%$ fetal bovine serum. The released cells were washed, pelleted, and seeded into tissue culture flasks.

\section{RNA Preparation and Quantitative Reverse Transcriptase-Polymerase Chain Reaction}

Total RNA was purified from aortic tissue and SMC cultures with TRIzol Reagents (Invitrogen, Carlsbad, Calif.). First-strand complementary DNA was synthesized with the Superscript II Reverse Transcriptase Kit (Invitrogen) and CD $3 \alpha$-specific reverse primers. The primers were 5'-CCCCAGAGGAAGCAAACCA-3' (forward) and 5'-TGGCCACCGACATCACATC-3' (reverse). Realtime polymerase chain reaction was performed with the QuantiTectTM SYBR Green PCR kit (QIAGEN Inc, Valencia, Calif) on ABI 7700 Real-Time PCR system (Applied Biosystems, Foster City, Calif.). $\beta$-Actin was used as the endogenous standard.

\section{TUNEL Testing}

TUNEL reaction was performed with an in situ cell detection kit (Promega Corporation, Madison, Wis) according to a standard protocol. Counterstaining for total nuclei was performed by mounting the sections with 4',6-diamidino-2'-phenylindole-containing glycerol solution. Total and TUNEL-positive nuclei were counted in 10 contiguous high-power fields under an Olympus fluorescent microscope (Olympus America Inc, Melville, NY) then converted to TUNEL-positive nuclei per square millimeter by two independent observers (R.H. and D.-C.G.).

\section{Immunohistochemical Testing}

The antibodies and supplier used are listed in Table E2, and biotin-conjugated antibodies (Vector Laboratories, Inc, Burlingame, Calif) were used as second antibody. An avidin-alkaline phosphatase-fast red reagent or the peroxidase-DAB system (Vectastain ABC kit; Vector Laboratories) was used to visualize the antibody stains. Normal mouse immunoglobulin G (Sigma, St Louis, Mo) served as the control for the immunostains. Infiltrated inflammatory cells were counted in 10 contiguous high-power 


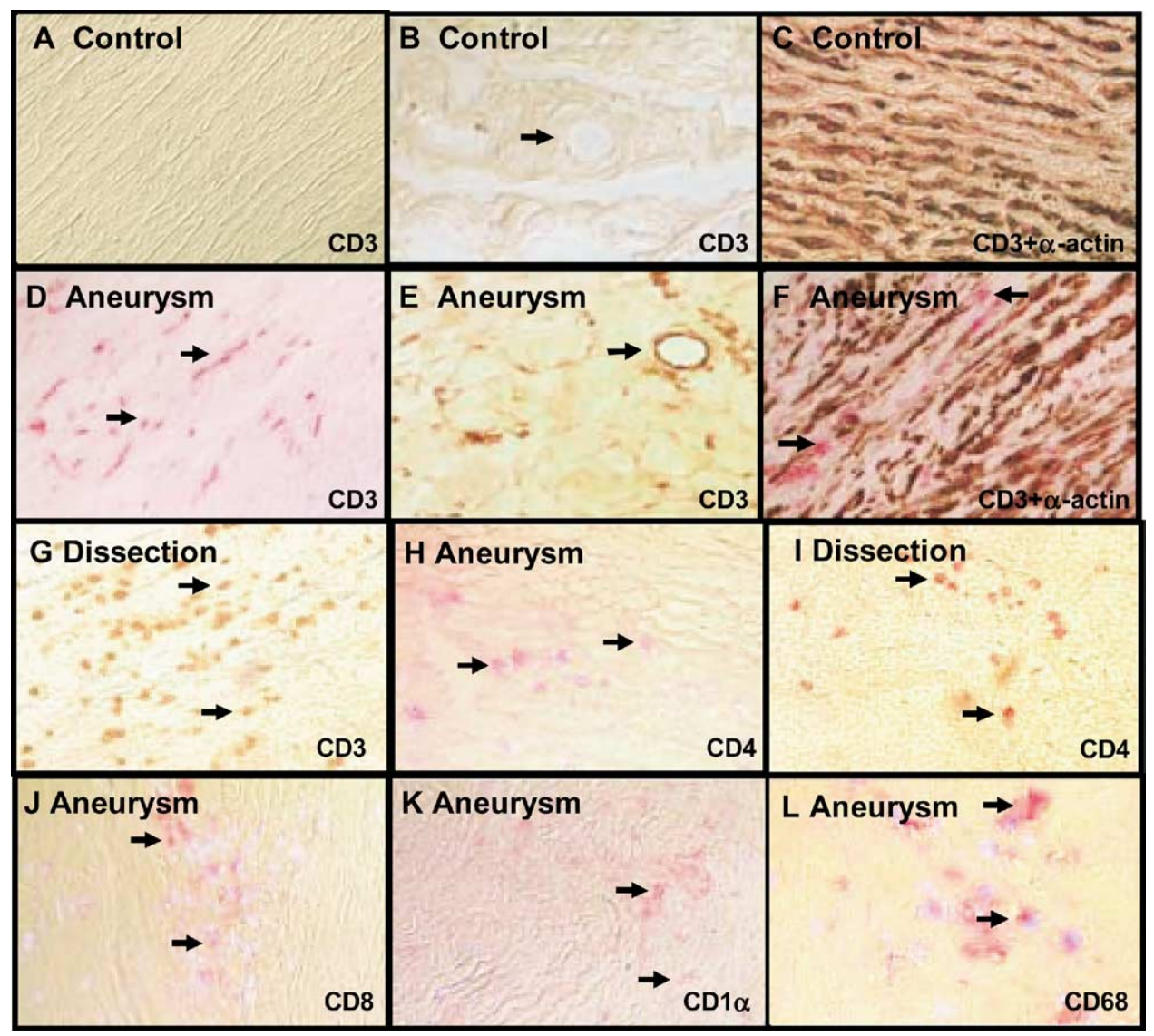

Figure 1. T-Iymphocyte infiltrates in TAA and TAD. For orientation, lumen of aorta is at top with adventitia at bottom. A through C, Immunohistochemical staining demonstrates that CD3 $^{+}$cells (arrow) are rarely present in control aorta. D, F, and G, Media from patients with TAA and TAD demonstrate CD3 $^{+}$cells (arrows) throughout media of aorta. $\mathrm{E}$, Adventitia from patient with TAA demonstrates $\mathrm{CD}^{+}$cells throughout adventitia. $\mathrm{H}$ through $\mathrm{J}_{\text {, }}$ $\mathrm{CD4}^{+}$and $\mathrm{CD8}^{+}$cells are present in aortic media from patients with TAA and TAD. $K$ and $\mathrm{L}$, Media from patient with TAA demonstrates that $\mathrm{CD} 1 \alpha^{+}$and $\mathrm{CD68}^{+}$cells are present in aneurysmal aortic media. Monoclonal anti-CD3 antibody used in D was obtained from DAKO Corporation, Carpinteria, Calif; antibodies in panels A through C and E through F were obtained from Zymed Laboratories Inc, South San Francisco, Calif, for comparison. Original magnification $400 \times$.

fields under an Olympus fluorescent microscope by two independent observers.

For double staining with TUNEL and immunostaining, the paraffin sections were stained by DeadEnd Fluorometric or Colorimetric TUNEL system (Promega Corporation, Madison, Wis), followed by immunohistochemical assay with an avidin-alkaline phosphatase-fast red reagent or the peroxidase-DAB system. The TUNEL-positive nuclei emitted green fluorescence when the DeadEnd Fluorometric TUNEL system was used or stained brown when the DeadEnd Colorimetric TUNEL System was used. Immunostaining for cell type-specific antigens showed pink color, which was clearly visible under a bright field microscope.

For assessing the amount of SMC $\alpha$-actin staining, the IT3 ImageTool (http://ddsdx.uthscsa.edu/dig/itdesc.html; Department of Dental Diagnostic Science, The University of Texas Health Science Center, San Antonio, Tex) was used to trace areas of positive $\alpha$-actin staining. The pixels of the area with positive $\alpha$-actin staining were quantified in a $10 \times$ magnification field for 5 patients with TAA, 6 with TAD, and 4 control patients.

\section{Statistical Analysis}

The CD3, TUNEL, and CD68 signals were counted in 10 contiguous high-power fields under an Olympus fluorescent microscope for each individual. The averaged values of these counts for each individual were used for later group comparisons. The 2-tailed Student $t$ test was conducted to compare the difference in mean values between study and control groups.

Further, the CD3, TUNEL, and CD68 values were treated as nonparametric data and expressed as median values. Mann-Whitney Wilcoxon rank-sum tests were used to compare groups on nonparametric data from CD3, TUNEL, and CD68. All tests of 
significance were 2-sided. All analyses were performed with SAS software (version 8.2; SAS Institute, Inc, Cary, NC).

\section{Results \\ Loss of SMCs and Elastic Fibers in Medial Degeneration}

The control aortas showed an orderly array of SMCs and elastic fibers in the media (Figure E1, A). In contrast, the aortas of the patients with TAAs and TADs showed medial degeneration affecting primarily the SMCs, leading to bands of cellular loss in the aortic media (Figure E1, B), regions with primarily the elastic fibers associated with increased basophilic ground substance, resulting in the appearance of cystlike structures (Figure E1,C), or both. ${ }^{10}$ Consistent with the hematoxylin and eosin staining results, immunostaining with monoclonal antibody against SMC $\alpha$-actin showed reduced numbers of SMC $\alpha$-actin-positive cells in medial bands in the diseased aortas, with focal loss of both SMCs and elastic fibers (Figure E1, $D$ through $F$ ). The amount of $\alpha$-actin staining was assessed quantitatively with a digital image analysis system. The amount of staining in histologic sections of control aortas (388,701 pixels) was 5-fold that in aneurysms $(73,417$ pixels, $P<.001)$ or dissected aortas $(75,906$ pixels, $P<.001)$.

\section{$\mathrm{CD3}^{+}$and $\mathrm{CD68}^{+}$Cells in the Aortas from Patients with TAA and TAD}

Immunohistochemical staining was used to determine whether inflammatory cells, specifically $\mathrm{T}$ cells and macrophages, were present in the aortic media of patients with TAAs with medial degeneration. Two different monoclonal anti-CD3 antibodies were used for pan-T lymphocyte detection, along with a monoclonal antibody against leukocyte common antigen (CD45) and a polyclonal antibody specific for macrophages (CD68). Rare $\mathrm{CD}^{+}$cells and macrophages were observed in the control aortas in the media or adventitia (Figure 1, $A$ through $C$ ). In the aortas of patients undergoing prophylactic repair of a TAA, there were significant increases in the number of $\mathrm{CD}^{+}$cells relative to control aortas $(P=.02$; Figures $1, D$ through $F$, and 2$) . \mathrm{CD}^{+}$cells were observed throughout the aortic media, either scattered throughout or localized in foci. Some $\mathrm{CD}^{+}$cells had the typical round appearance of lymphocytes, but other $\mathrm{CD} 3^{+}$ cells had a flattened morphology similar to SMCs (Figure 1, $D$, arrows). The $\mathrm{CD}^{+}$cells were present in similar abundance when a different monoclonal antibody against CD3 was used for staining. Interestingly, there was a considerable influx of $\mathrm{CD}^{+}$cells in the walls of the vasa vasorum in the adventitia that was not present in control aortas (Figure 1, $B$ and $E$ ). Staining of endothelial cells with an antibody directed against Von Willebrand factor verified that the $\mathrm{CD}^{+}$cells were associated with the vasa vasorum in the adventitia (data not shown). The presence of $\mathrm{CD}^{+}$ cells was confirmed by immunostaining with an antibody to

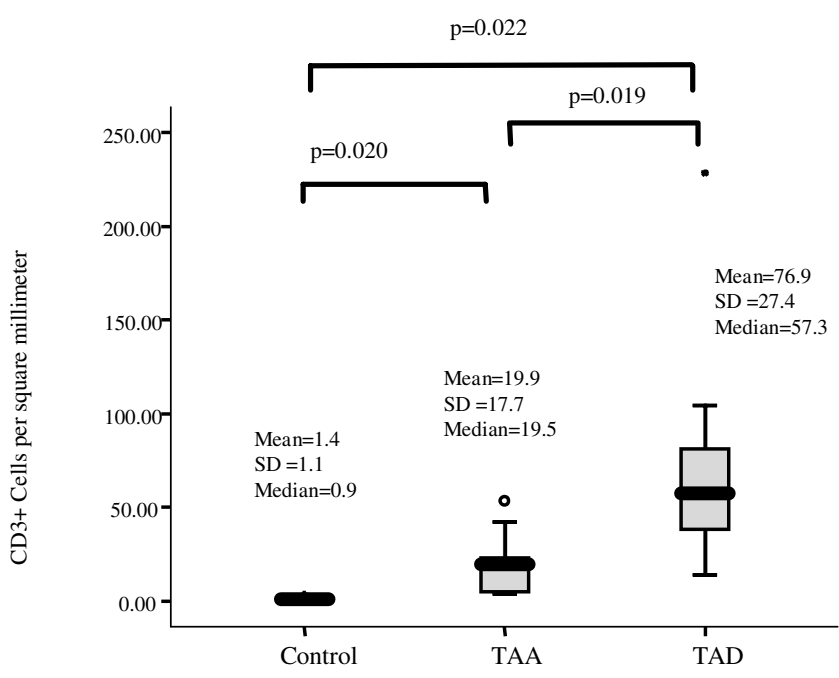

Figure 2. $\mathrm{CD3}^{+}$cells present in media from control, aneurysmal, and dissected aortas. $\mathrm{CD3}^{+}$cells in $1 \mathrm{~mm}^{2}$ of aortic media were counted from aortic tissue sections from control subjects, patients with TAAs, and patients with TADs. Total areas examined were 2.2 mm$^{2}$. Box-whisker plots display CD3. Central horizontal bars indicate median, upper and lower ends of boxes indicate 75th and 25th percentiles, and error bars indicate maximum and minimum values of CD3 values. $P$ values were determined by Mann-Whitney Wilcoxon rank-sum tests.

CD45, an antigen present on all lymphocytes. CD45 ${ }^{+}$cells had a similar distribution to that of the $\mathrm{CD}^{+}$cells in the medial layer.

Focal and diffuse accumulations of $\mathrm{CD}^{+}$cells were detected in aortas of patients with TADs, and the numbers of these cells were increased relative to control aortas $(P=$ $.022)$ and to aneurysmal aortas $(P=.019$; Figures $1, G$, and 2$)$. $\mathrm{CD}^{+}$cells were observed both along the edges of ruptured media of the dissected aortas and distributed throughout the media with a flattened morphology.

To assess the T-cell subsets within aortic lesions, the sections were stained with antibodies that recognized CD4 and CD8 antigens, markers for helper and cytotoxic T cells, respectively. Both $\mathrm{CD}^{+}{ }^{+}$and $\mathrm{CD} 8^{+}$T-cell subsets were presented in the aneurysmal and dissected aortas but not in control aortas (Figure 1, $H$ through $J$ ). Immunostaining with antibodies directed against $\mathrm{CD} 1 \alpha$ antigen indicated that vascular dendritic cells were frequently observed in the areas containing a large number of $\mathrm{T}$ cells but were not found in control aortas (Fig. 1K). Finally, immunostaining with CD68 antibody also indicated that macrophages were frequently present in the aortas from patients with ascending aortic aneurysm and dissection (Fig. 1L).

The flattened appearance of the CD3 + cells raised the question as to whether some SMCs were expressing the CD3 antigen, and double staining using monoclonal anti- 

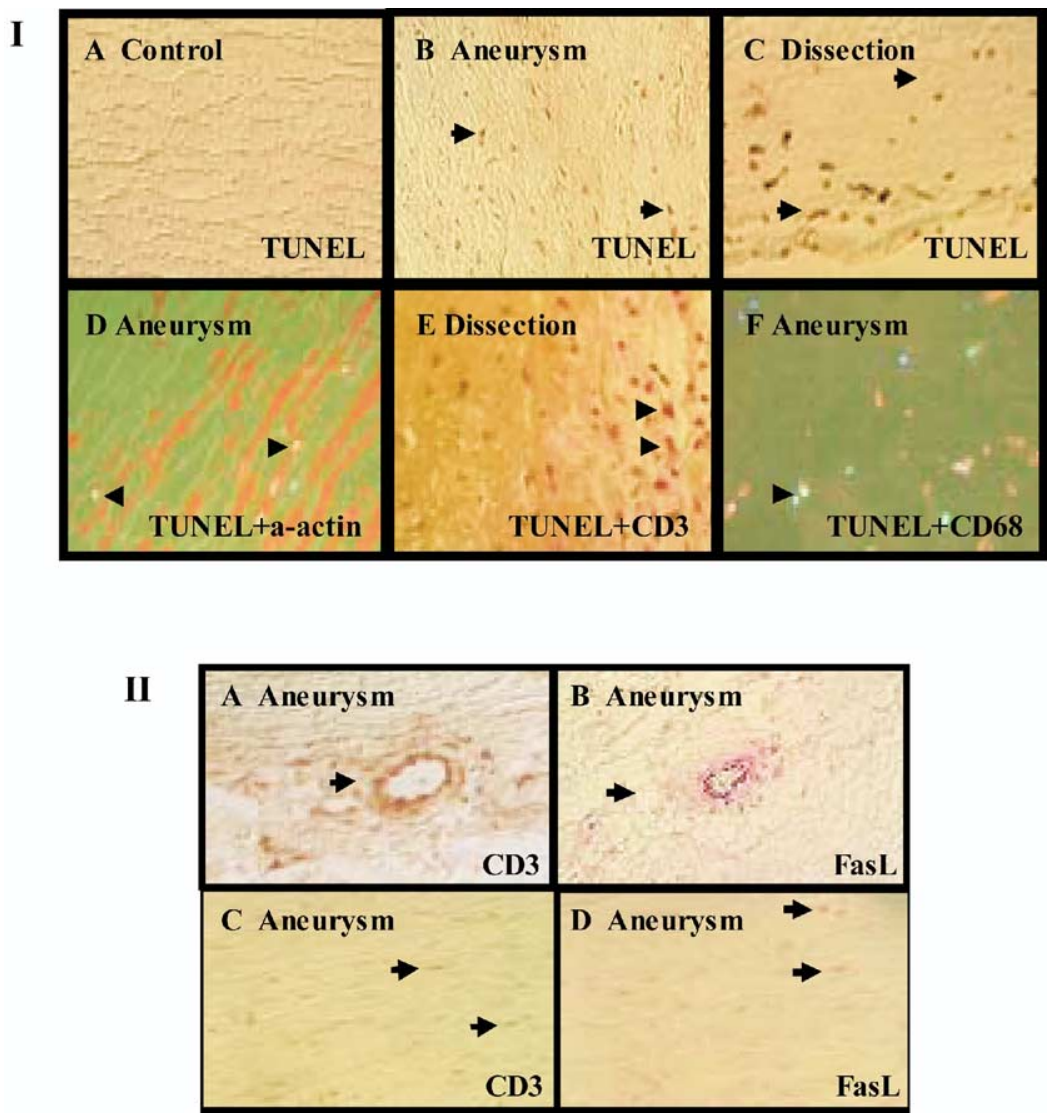

Figure 3. Increased TUNEL-positive and FasL staining in media of aneurysmal and dissected aortas. Top: A, TUNEL-positive cells (arrows) are rarely present in control aorta. B and C, Media from patients with TAA and TAD demonstrated TUNEL-positive cells (arrows). D through $F$, Double staining with combination of TUNEL and immunohistochemical assay with $\alpha$-actin, CD3, and CD68 antibodies demonstrated that SMCs, T lymphocytes, and macrophages were undergoing apoptosis (arrows) in aortic media from patient with TAA. TUNEL staining in panels A through $C$ and $E$ used DeadEnd Colorimetric TUNEL System; that in panels D and $F$ used DeadEnd Fluorescence TUNEL System. Bottom: A and C, Adventitia and media in aneurysmal aorta indicate influx of $\mathrm{CD3}^{+}$cells in walls of vasa vasorum and aortic media. $B$ and $D$, Same vasa vasorum and media were stained with anti-FasL antibody, which indicated colocation of $\mathrm{CD3}^{+}$and FasL-positive cells. Magnification $400 \times$.

bodies against CD3 and SMC $\alpha$-actin was done to address this question. The double staining revealed that the majority of cells positive for SMC $\alpha$-actin were distinct from those staining for CD3 (Fig. 1C and G). However, in certain regions with leukocyte accumulation, an overlap of CD3 and SMC $\alpha$-actin immunostaining was observed. To further address this question, total RNA was isolated from control and aneurysmal aortas and explanted SMC cultures from patients with TAA, and messenger RNA (mRNA) expression of the $\mathrm{CD} 3 \alpha$ polypeptide was determined with quantitative reverse transcriptase-polymrase chain reaction. Consistent with the results of immunohistochemical staining, we detected $\mathrm{CD} 3 \alpha$ mRNA in the total mRNA isolated from aortas of patients with TAAs but not from mRNA from the control aortas. CD $3 \alpha$ mRNA was not detected in the total
mRNA isolated from SMC cultures explanted from aneurysmal aortas, providing further data that the SMCs are not expressing CD $3 \alpha$.

Immunohistochemical testing with a polyclonal antibody directed against CD68 was used to determine whether macrophages were also present in the diseased aortic tissue. Numbers of macrophages were significantly increased in the media in both aneurysmal $\left(5.5 \pm 1.8\right.$ cells $\left./ \mathrm{mm}^{2}, P=.01\right)$ and dissected $\left(6.0 \pm 1.4\right.$ cells $\left./ \mathrm{mm}^{2}, P=.005\right)$ aortas relative to control aortas $\left(0.7 \pm 0.2 \mathrm{cells} / \mathrm{mm}^{2}\right)$.

\section{TAAs and TADs Exhibit Increased Markers of Apoptosis}

To test the hypothesis that infiltration of $\mathrm{T}$ lymphocytes leads to activation of the death-promoting pathways through 


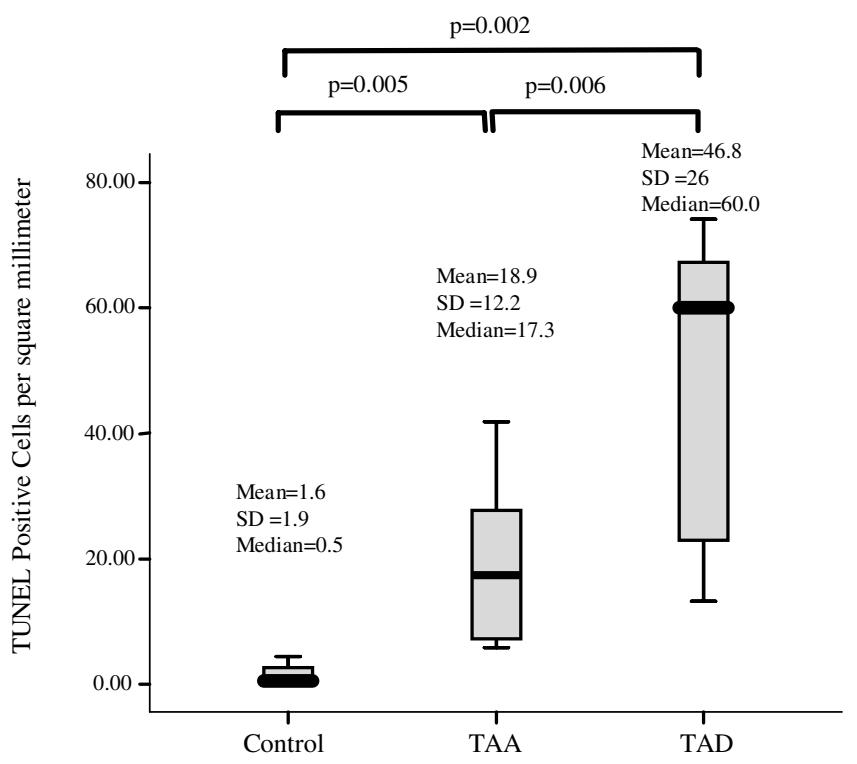

Figure 4. TUNEL-positive cells present in media from control, aneurysmal, and dissected aortas. TUNEL-positive cells in $1 \mathrm{~mm}^{2}$ of the aortic media were counted from aortic tissue sections from control patients, patients with TAAs, and patients with TADs. Total areas examined were $2.2 \mathrm{~mm}^{2}$. Box-whisker plots display TUNEL. Central horizontal bars indicate median, upper and lower ends of boxes indicate 75th and 25th percentiles, and error bars indicate maximum and minimum values of TUNEL values. $P$ values were determined by Mann-Whitney Wilcoxon rank-sum tests.

Fas-FasL engagement and caspase activation, DNA fragmentation was initially analyzed on the aortic specimens with the TUNEL assays. Aortas from patients without aortic disease showed few TUNEL-positive cells (Figures 3, A top, and 4). In contrast, TUNEL-positive cells were present in aortas from both the TAA group $(P=.005)$ and the TAD group $(P=.002)$ at significantly higher levels than in control aortas, with significantly higher numbers of TUNEL-positive cells in the dissected aortas (Figures 3, A top through $C$ top, and 4). The TUNEL-positive cells were primarily distributed in the regions with elastic fibril loss, and there was focal accumulation at the edges of ruptured media in the dissected aortas (Figure 3, C top). Double immunostaining with a combination of TUNEL and immunohistochemical techniques confirmed the colocation of TUNEL-positive cells with SMCs, lymphocytes, and macrophages (Figure 3, $D$ top through $F$ top).

Immunohistochemical testing demonstrated diffuse staining of immunoreactive Fas antigens throughout the media and adventitia of the control aorta, with increased amounts in both locations in the diseased aortas (data not shown). In contrast, staining for FasL was mainly associated with cells in the adventitia, in particular around the vasa vasorum, with occasional positive cells in the media of aneurysmal and dissected aortas colocated with the $\mathrm{CD} 3^{+}$cells (Figure 3, A bottom through $D$ bottom). Consistent with the results obtained with immunohistochemical testing, increased levels of Fas and FasL antigen were detected in the aneurysmal and dissected tissues relative to control aortas (Figure E2). In contrast, the control aortas displayed modest expressions of Fas and FasL immunoreactivity. Furthermore, both the aneurysmal and dissected aortas showed immunoreactivity to activated caspase-3, whereas control aortas showed almost no activated caspase-3 (Figure E2).

\section{Discussion}

Medial degeneration associated with TAAs and TADs was originally described by Erdheim ${ }^{1}$ as a noninflammatory lesion associated with loss of SMCs and elastic fibers in the media. Evidence presented in this report indicates that $\mathrm{T}$ lymphocytes and macrophages are common features in the aortas of patients with medial degeneration. Immunohistochemical testing with two different monoclonal antibodies directed against CD3 clearly showed the presence of $\mathrm{T}$ lymphocytes in the media, either diffusely throughout the media or in focal accumulations. Some of the $\mathrm{CD}^{+}$cells with flattened appearance in the aortic media resided between the SMC layers, suggesting a close interaction between these inflammatory cells and the vascular cells. Flattened perforin-positive cells, presumably $\mathrm{T}$ lymphocytes, were previously observed in AAAs, indicating that lymphocytes may assume this morphologic appearance in the aortic media. ${ }^{11}$ The infiltration of $\mathrm{CD}^{+}$cells in the walls of the vasa vasorum in this study suggests that the $T$ cells might migrate from these blood vessels of the adventitia into the media of the aortas. This study also demonstrated increased $\mathrm{CD}{ }^{+}$cells in the diseased aortas, indicating the presence of macrophages in this lesion.

A number of previous studies have documented evidence of increased apoptosis of SMCs in medial degeneration. ${ }^{12,13}$ Immunohistochemical testing has indicated that medial degeneration of aorta is associated with p53 accumulation, Bax upregulation, and apoptosis of SMC in the aortas of patients with TAD. ${ }^{14}$ Our study showed an increase in the amount of apoptotic cells by TUNEL staining in the media of both aneurysmal and dissected aortas. Double staining for TUNEL-positive nuclei and cell-specific markers provides evidence that SMCs are undergoing apoptosis, and to a lesser extent are lymphocytes and macrophages. Immunohistochemical results indicate colocation of FasL staining with CD3 staining, suggesting that $\mathrm{T}$ lymphocytes were the cells responsible for FasL production. In contrast, Fas was found diffusely throughout the media and adventitia. These results suggest apoptosis of primarily SMCs through the Fas-FasL pathway in aneurysmal tissue. The relative lack of evidence that substantial numbers of $\mathrm{CD}^{+}$cells are undergoing apoptosis suggests that the aorta is not an immune- 
privileged site in which the Fas produced by SMCs leads to apoptosis of $\mathrm{T}$ lymphocytes to limit an inflammatory reaction.

The $\mathrm{T}$ lymphocyte and macrophage infiltration and TUNEL staining were more pronounced in the media of the dissected aortas than of the aneurysmal aortas. The dissection of blood into the wall of the aorta is a traumatic tissue injury, and it is therefore not surprising that inflammatory markers and apoptotic markers would be increased in the damaged tissue. Although both inflammatory and TUNELpositive cells were observed along the margin of the dissections, the inflammatory cells and apoptotic markers were also present throughout the media. It is possible that the degree of inflammation and apoptosis in the media may be increased in an aorta before dissection. These events could potentially lead to increased rates of loss of SMCs and destruction of the tissue, setting the stage for the dissection to occur. The current recommendation is to prophylactically repair an aneurysm involving the ascending aorta to prevent dissection when it is larger than $5 \mathrm{~cm}$ in diameter or when the aorta is rapidly enlarging. ${ }^{15}$ Some have dissection when the aorta is minimally enlarged, however, and future studies will determine whether this premature dissection is related to the degree of inflammation and apoptosis in the aortic media.

Inflammation producing enzymatic remodeling of the vascular extracellular matrix is emerging as a shared pathologic mechanism in a variety of vascular diseases, including atherosclerosis, AAAs, and intracranial aneurysms. AAAs are characterized by atherosclerosis leading to a thinning of the tunica media and rarefaction of SMCs. ${ }^{11,16}$ Many medial SMCs in AAAs bear markers of apoptosis and signals capable of initiating cell death, implying that apoptotic death may contribute to the reduction of cellularity and impaired matrix homeostasis. $\mathrm{CD} 4^{+}$and $\mathrm{CD} 8^{+} \mathrm{T}$ cells are also present in AAAs, and these cells have been shown to express cytotoxic mediators, including Fas and perforin, which causes death of T cell-targeted cells. The contribution to elimination of SMCs of death-promoting products of activated immune cells was suggested by the observations made in this study of TAAs and TADs associated with medial degeneration. Thus this study provides new evidence that inflammatory infiltrate of $\mathrm{T}$ cells expressing Fas may contribute to TAAs in a similar fashion as to AAAs.

It is important to acknowledge the limitations of a study that uses surgically removed human aneurysmal aortas. TAAs were not removed until the diameter of the aorta reached 5.0 to $5.5 \mathrm{~cm}$, and TAD represents an acute traumatic insult to the aorta. Therefore these studies involved end-stage tissues for both disease processes. Furthermore, the patients were placed on cardiopulmonary bypass and hypothermic circulatory arrest and treated with aprotinin to limit inflammation, and we cannot exclude the possibility that these procedures induced inflammatory cells in the aortic wall. The results presented on these tissues cannot be extrapolated to earlier phases of aneurysm formation. The development of homozygous mice with a hypomorphic $f b n l$ allele as an animal model of Marfan syndrome has shed some insight into the role of inflammation in the pathogenesis of aneurysms and dissections. ${ }^{17,18}$ Homozygous mice die at a few months of age, with half of the deaths resulting from vascular rupture. A surprising observation in these mice is the presence of a monocytic infiltration of the medial layer, evident from 8 weeks on that correlated with fragmentation of elastic lamellae, loss of elastin content, and dilation of the vessel wall. Thus the only mouse model of TAA and TAD also demonstrates an inflammatory infiltrate in the aortic wall.

In summary, the data in this study provide the first evidence that medial degeneration of the aorta, originally described by Erdheim, ${ }^{1}$ is associated with T-lymphocyte and macrophage infiltration into the media of the aortic wall. Immunoblot and immunohistochemical analyses suggest that death-promoting signaling factors from the T lymphocytes may contribute to SMC apoptosis. In addition to apoptosis of the SMCs, evidence is emerging that the SMCs have an altered phenotype, which includes increased synthesis of matrix metalloproteinases and tissue inhibitors of metalloproteinases. An imbalance between matrix metalloproteinases and tissue inhibitors of metalloproteinases in the tissue may lead to elastic fiber degradation, further decreasing the anchoring of the SMCs to these matrix structures. A more detailed pathogenesis for medial degeneration is emerging, with an altered SMC phenotype, degradation of the elastic fibers, T-cell infiltration, and apoptosis of the SMCs playing roles in this pathologic lesion.

\section{References}

1. Erdheim J. Medionecrosis aortae idiopathica cystica. Virchows Arch Path Anat. 1930;276:187-229.

2. Carlson RG, Lillehei CW, Edwards JE. Cystic medial necrosis of the ascending aorta in relation to age and hypertension. Am J Cardiol. 1970;25:411-5.

3. Schlatmann TJ, Becker AE. Histologic changes in the normal aging aorta: implications for dissecting aortic aneurysm. Am J Cardiol. 1977;39:13-20.

4. Silverman DI, Gray J, Roman MJ, Bridges A, Burton K, Boxer M, et al. Family history of severe cardiovascular disease in Marfan syndrome is associated with increased aortic diameter and decreased survival. J Am Coll Cardiol. 1995;26:1062-7.

5. Dietz HC, Pyeritz RE. Mutations in the human gene for fibrillin-1 (FBN1) in the Marfan syndrome and related disorders. Hum Mol Genet.1995;4Spec No:1799-809.

6. Milewicz DM, Urban Z, Boyd C. Genetic disorders of the elastic fiber system. Matrix Biol. 2000;19:471-80.

7. Girardi LN, Coselli JS. Inflammatory aneurysm of the ascending aorta and aortic arch. Ann Thorac Surg. 1997;64:251-3.

8. Biddinger A, Rocklin M, Coselli J, Milewicz DM. Familial thoracic aortic dilatations and dissections: a case control study. J Vasc Surg. 1997;25:506-11.

9. Roth M, Lemke P, Bohle RM, Klovekorn WP, Bauer EP. Inflammatory aneurysm of the ascending thoracic aorta. J Thorac Cardiovasc Surg. 2002;123:822-4. 
10. Gould SE. Pathology of the heart and blood vessels. 3rd ed. Springfield (III): Charles C Thomas; 1968.

11. Schonbeck U, Sukhova GK, Gerdes N, Libby P. T(H)2 predominant immune responses prevail in human abdominal aortic aneurysm. Am J Pathol. 2002;161:499-506.

12. Lopez-Candales A, Holmes DR, Liao S, Scott MJ, Wickline SA, Thompson RW. Decreased vascular smooth muscle cell density in medial degeneration of human abdominal aortic aneurysms. Am J Pathol. 1997;150:993-1007.

13. Bonderman D, Gharehbaghi-Schnell E, Wollenek G, Maurer G, Baumgartner H, Lang IM. Mechanisms underlying aortic dilatation in congenital aortic valve malformation. Circulation. 1999;99:2138-43.

14. Ihling C, Szombathy T, Nampoothiri K, Haendeler J, Beyersdorf F, Uhl M, et al. Cystic medial degeneration of the aorta is associated with p53 accumulation, Bax upregulation, apoptotic cell death, and cell proliferation. Heart. 1999;82:286-93.
15. Gott VL, Greene PS, Alejo DE, Cameron DE, Naftel DC, Miller DC, et al. Replacement of the aortic root in patients with Marfan's syndrome. N Engl J Med. 1999;340:1307-13.

16. Henderson EL, Geng YJ, Sukhova GK, Whittemore AD, Knox J, Libby P. Death of smooth muscle cells and expression of mediators of apoptosis by $\mathrm{T}$ lymphocytes in human abdominal aortic aneurysms. Circulation. 1999;99:96-104.

17. Bunton TE, Biery NJ, Myers L, Gayraud B, Ramirez F, Dietz HC. Phenotypic alteration of vascular smooth muscle cells precedes elastolysis in a mouse model of Marfan syndrome. Circ Res. 2001; 88:37-43.

18. Pereira L, Lee SY, Gayraud B, Andrikopoulos K, Shapiro SD, Bunton $\mathrm{T}$, et al. Pathogenetic sequence for aneurysm revealed in mice underexpressing fibrillin-1. Proc Natl Acad Sci U S A. 1999;96: 3819-23.

\section{Authoritative}

The Journal of Thoracic and Cardiovascular Surgery is the most frequently cited thoracic/cardiovascular surgery journal in the Science Citation Index. An article in JTCVS is cited on average almost twice as often as those in the closest cardiothoracic journal. 
TABLE E1. Clinical information of individuals participating in the study

\begin{tabular}{|c|c|c|c|c|c|c|}
\hline Sample & Group & Age (y) & Sex & Hypertension & Smoker & Aortic diameter (cm) \\
\hline 2613 & Control* & 44 & Male & NA & NA & NA \\
\hline 2664 & Controlt & 48 & Female & NA & NA & NA \\
\hline 2701 & Controlł & 54 & Female & NA & NA & NA \\
\hline 2840 & Control§ & 74 & Female & NA & NA & NA \\
\hline 2976 & Control $^{2}$ & 66 & Male & NA & NA & NA \\
\hline 2478 & Aneurysm & 74 & Female & Yes & Yes & 5.6 \\
\hline 2496 & Aneurysm & 77 & Female & Yes & No & 5.9 \\
\hline 2761 & Aneurysm & 49 & Male & Yes & Yes & 5.0 \\
\hline 2762 & Aneurysm & 65 & Female & Yes & Yes & 6.0 \\
\hline 2945 & Aneurysm & 40 & Female & No & No & 5.0 \\
\hline 2955 & Aneurysm & 52 & Male & Yes & No & 5.2 \\
\hline 3386 & Aneurysm & 74 & Female & Yes & No & 7.0 \\
\hline 3387 & Aneurysm & 64 & Female & Yes & Yes & 5.8 \\
\hline 3393 & Aneurysm & 71 & Male & Yes & Yes & 5.6 \\
\hline 2482 & Dissection & 71 & Female & Yes & No & 5.7 \\
\hline 2789 & Dissection & 59 & Male & No & Yes & 4.5 \\
\hline 2813 & Dissection & 55 & Female & Yes & No & 5.5 \\
\hline 2992 & Dissection & 62 & Female & Yes & No & 8.0 \\
\hline 2993 & Dissection & 57 & Male & Yes & Yes & 5.2 \\
\hline 3038 & Dissection & 56 & Male & Yes & Yes & 4.3 \\
\hline 3418 & Dissection & 59 & Male & No & No & 3.8 \\
\hline
\end{tabular}

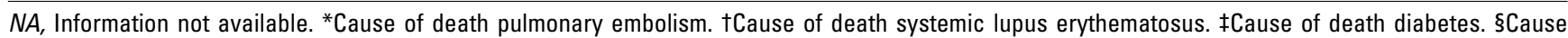
of death stroke. ${ }^{2}$ Cause of death congestive heart disease.

TABLE E2. Antibodies used for immunohistochemical and immunoblot analyses

\begin{tabular}{|c|c|c|c|c|}
\hline Antibody & Cell type & Source & Manufacturer & Concentration \\
\hline SMC $\alpha$-acting & SMC & Mouse monoclonal & Sigma & 1:50 IHC \\
\hline CD3 & Pan T cell & Mouse monoclonal & DAKO & 1:25 IHC \\
\hline CD3 & Pan T cell & Mouse monoclonal & Zymed & $1: 1 \mathrm{IHC}$ \\
\hline CD4 & Helper T cell & Mouse monoclonal & DAKO & 1:20 IHC \\
\hline CD8 & Cytotoxic T cell & Mouse monoclonal & DAKO & 1:20 IHC \\
\hline CD1a & Dendritic cell, Langerhans cell & Mouse monoclonal & BD PharMingen & $1: 100 \mathrm{IHC}$ \\
\hline Interleukin 4 & $\mathrm{~T}_{\mathrm{H}} 2$ cell & Mouse monoclonal & R\&D Systems & $1: 100 \mathrm{IHC}, 1 \mathrm{mg} / \mathrm{mL} \mathrm{IB}$ \\
\hline Interleukin 10 & $\mathrm{~T}_{\mathrm{H}} 2$ cell & Goat & R\&D Systems & $1: 1000 \mathrm{IHC}, 0.1 \mathrm{mg} / \mathrm{mL} \mathrm{IB}$ \\
\hline Interferon $\gamma$ & $\mathrm{T}_{\mathrm{H}} 1$ cell & Mouse monoclonal & BD PharMingen & $1 \mathrm{mg} / \mathrm{mL} \mathrm{IB}$ \\
\hline CD45 & Leukocyte & Mouse monoclonal & Santa Cruz* & $1: 100 \mathrm{IHC}$ \\
\hline CD68 & Macrophage & Rabbit polyclonal & Santa Cruz* & $1: 100 \mathrm{IHC}$ \\
\hline Fas & Inflammatory cell, SMC & Mouse monoclonal & BD PharMingen & 1:100 IHC, $1 \mathrm{mg} / \mathrm{mL} \mathrm{IBt}$ \\
\hline FasL & T cell, macrophage & Mouse monoclonal & BD PharMingen & 1:100 IHC, $1 \mathrm{mg} / \mathrm{mL}$ IB \\
\hline Caspase-3 & Various & Rabbit polyclonal & BD PharMingen & $1: 100 \mathrm{IHC}, 0.5 \mathrm{mg} / \mathrm{mL} \mathrm{IB}$ \\
\hline
\end{tabular}

$I H C$, Immunohistochemical testing; $I B$, immunoblot testing. *Santa Cruz Biotechnology, Inc, Santa Cruz, Calif. 


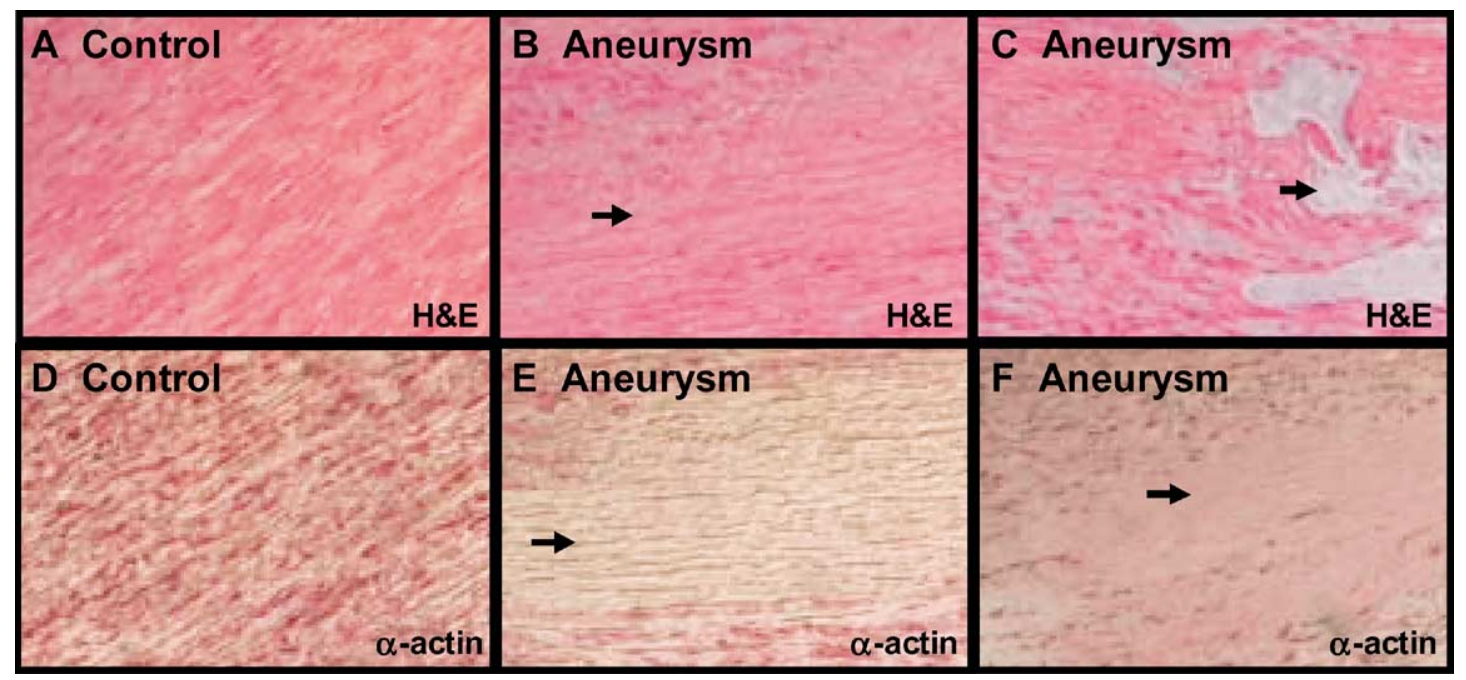

Figure E1. Medial degeneration of aorta associated with loss of SMCs and elastic fibers. Representative cross-sections of human control and aneurysmal aortic tissue were stained with hematoxylin and eosin (A-C) or with monoclonal antibody directed against SMC $\alpha$-actin (D-F). A and D, Media from control aorta demonstrates nuclei and elastic fibers throughout media. $B$ and $E$, Media from patient with TAA reveals loss of cell nuclei in band in media, but elastic fibers remain. $C$ and $F$, Media in different field from same patient as in $B$ and $E$ displays areas with loss of both cells and nuclei. Magnification 100x.

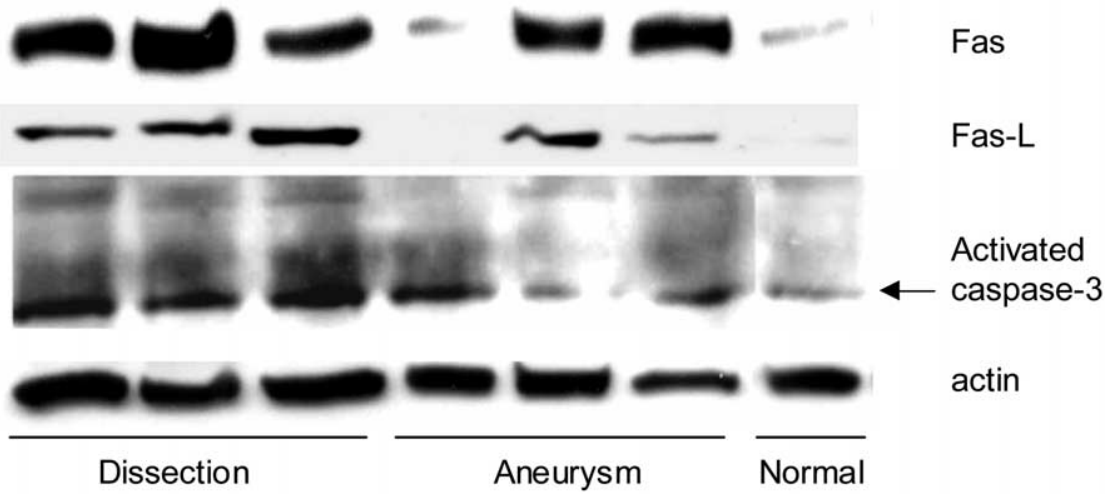

Figure E2. Immunoblot analysis showed levels of Fas, FasL, and activated caspase-3 to be increased in aneurysmal and dissected aortas. Levels of Fas and FasL were assessed relative to actin. Increased levels of Fas antigen were detected in aneurysmal $(0.82 \pm 0.39)$ and dissected $(0.68 \pm 0.62)$ tissues relative to control tissues $(0.07)$. Increased levels of FasL antigen were detected in aneurysmal $(0.71 \pm 0.23)$ and dissected $(0.42 \pm 0.31)$ tissues relative to control tissues (0.15). Total protein loaded into each lane was $50 \mu \mathrm{g}$. After separation by sodium dodecylsulfatepolyacrylamide gel electrophoresis, membranes were blocked and incubated with primary antibody against Fas, FasL, and activated caspase-3. Membranes were incubated with horseradish peroxidase-linked secondary antibodies, followed by visualization with SuperSignal West Femto kit (Pierce Biotechnology, Inc, Rockford, III). Bands correspond to following molecular weighs of proteins: Fas, 40 kd; FasL, 37 kd; activated caspase-3, 20.4 kd. Results are consistent with results obtained with immunohistochemical testing. 